\title{
High Magnetic Field Detunes Vibronic Resonances in Photosynthetic Light Harvesting
}

Margherita Maiuri ${ }^{1}$, Maria B. Oviedo ${ }^{1,2,3}$, Jacob C. Dean ${ }^{1}$, Michael Bishop ${ }^{4}$, Bryan Kudisch ${ }^{1}$, Zi. S. D. Toa ${ }^{1}$, Bryan M. Wong ${ }^{2}$, Stephen A. McGill ${ }^{4}$, Gregory D. Scholes ${ }^{1 *}$

${ }^{1}$ Department of Chemistry, Princeton University, Washington Road, Princeton, New Jersey 08540, United States.

${ }^{2}$ Department of Chemical \& Environmental Engineering and Materials Science \& Engineering Program, University of California-Riverside, Riverside, California 92521, United States.

${ }^{3}$ Instituto de Investigaciones Fisicoquímicas de Córdoba, Consejo Nacional de Investigaciones Científicas y Técnicas (INFIQC - CONICET), Departamento de Química Teórica y Computacional, Facultad de Ciencias Químicas, Universidad Nacional de Córdoba, Ciudad Universitaria, Córdoba X5000HUA, Argentina.

${ }^{4}$ National High Magnetic Field Laboratory (NHFML), 1800 E. Paul Dirac Drive, Tallahassee, Florida, 32310, United States.

DOI: $10.1021 /$ acs.jpclett. 8 b02748

\section{Corresponding Author}

*Correspondence to: gscholes@princeton.edu 
ABSTRACT: The origin and role of oscillatory features detected in recent femtosecond spectroscopy experiments of photosynthetic complexes remain elusive. A key hypothesis underneath these observations relies on electronic-vibrational resonance, where vibrational levels of an acceptor chromophore match the donor-acceptor electronic gap, accelerating the downhill energy transfer. Here we identify and detune such vibronic resonances using a high magnetic field that exclusively shifts molecular exciton states. We implemented ultrafast pump-probe spectroscopy into a specialized 25 Tesla magnetic field facility and studied the light-harvesting complex PC645 from a cryptophyte algae where strongly coupled chromophores form molecular exciton states. We detect a change in high-frequency coherent oscillations when the field is engaged. Quantum chemical calculations coupled with a vibronic model explain the experiment as a magnetic field-induced shift of the exciton states which in turn affects the electronicvibrational resonance between pigments within the protein. Our results demonstrate the delicate sensitivity of inter-pigment coherent oscillations of vibronic origin to electronic-vibrational resonance interactions in light harvesting complexes.

Reports of coherent beatings in complex systems continue to attract researchers because they suggest a mechanism for tuning photo-induced reactions, e.g. energy transfer, where possible implications have not previously been deeply considered ${ }^{1}$. Theoretical studies have now predicted that coherent oscillations observed in many ultrafast experiments on light-harvesting complexes $^{2-5}$ are assigned to vibronic coherence-quantum mechanical excitation delocalization that depends on the nuclear coordinates of the light-harvesting chromophores ${ }^{6-15}$. This means that 
the vibrational motion of the chromophores involved becomes correlated by electronic coupling, so the ladders of states of the chromophores do not independently absorb or emit energy. Thus, in many cases the long lived coherent oscillations observed in light-harvesting complexes (and charge separation ${ }^{16}$ ) can be explained by exciton-vibration resonance.

Resonance effects are in general of significant importance in photo-induced chemical reactivity because they have pronounced functional consequences. For example, radiationless transitions (e.g. internal conversion) are dictated by the energy gap law, where resonantenhancement of the rate leads to its exponential dependence on the energy gap between the initial and final state ${ }^{17}$. "Flickering resonances" in bridge-mediated electron transfer along DNA strands are predicted to bring dynamically disordered intermediate states into electronic degeneracy, supporting band-like electron transport during a short transient resonance time ${ }^{18}$. The significance of resonance enhancement in photochemistry calls for incisive experimental methods to measure electronic-vibrational resonances and clarify their implication in photosynthetic energy transfer. Recent studies employing two-dimensional electronic spectroscopy (2DES) suggested the presence suggested the presence of electronic-vibrational mixed coherences in various photosynthetic complexes ${ }^{16,20,21}$. However, an unequivocal identification of the vibronic coupling and its sensitivity to resonance conditions is a challenging task owing to the complex structure of 2DES signals. A suitable experiment should remove the resonance between the vibrational frequency and the electronic frequency difference between donor and acceptor chromophores and detect the concomitant intensity and energy redistribution initially caused by vibronic mixing. A possible way to remove the electronic resonance could be to exploit protein mutagenesis in such a way as to energetically shift the absorption origin of one chromophore $^{22}$, however a less 'invasive' and controllable method is desirable. 
Here we demonstrate how a strong external magnetic field can be used to detune vibronic resonance in the light harvesting complex PC645 isolated from the aquatic cryptophyte algae Chroomonas mesostigmatica (CCMP 269). The idea of using an external magnetic field to perturb electronic transitions rely on spin-orbit coupling. An external magnetic field interacts with a molecule through the electronic part of its wavefunction and has two primary effects on the observed energy levels: a spin splitting of electronic levels (Zeeman splitting) and a diamagnetic shift, an increase in energy of both of the spin-split levels with magnetic field. In a diamagnetic system such as PC645, small static magnetic fields negligibly perturb the molecular absorption spectrum. However, if the magnetic energy becomes comparable to the excitonic coupling (typically at magnetic fields of several Tesla), these fields start to affect the spatial distribution of the orbital electronic wavefunctions of electrons involved in the photoexcitation. We also assume that the nuclear motion, and hence vibrational spectrum, of a molecule (that is not freely rotating) will not be perturbed by the magnetic field, since the magnetic moment generated by the atomic motion is negligible ${ }^{23}$. Thus, a high magnetic field can act as a noninvasive reagent for disentangling electronic/vibrational contributions to interchromophoric interactions.

\section{Calculated magnetic field effect on PC645 light-harvesting complex}

The Chroomonas PC645 light harvesting complex comprises four chromophore pairs (fig. 1a) which are responsible for the broad absorption in the yellow-orange part of the solar spectrum. These chromophores establish an efficient energy funnel from the core to the periphery of the complex ${ }^{24}$. A prominent pathway involved in the function of this protein is excitation of the highest energy band, at $\sim 17000 \mathrm{~cm}^{-1}$, of an exciton state where the energy is 
coherently delocalized across two dihydrobiliverdin (DBV) chromophores in the center of the light-harvesting complex ${ }^{25}$. A direct energy transfer channel from the donor lowest excitonic state $\left(\mathrm{DBV}^{-}\right)$to the peripheral phycocyanobilin (PCB) acceptors (fig. 1b) - absorbing below $15500 \mathrm{~cm}^{-1}-$ dominates and energy is transferred in about $600 \mathrm{fs}^{24}$.

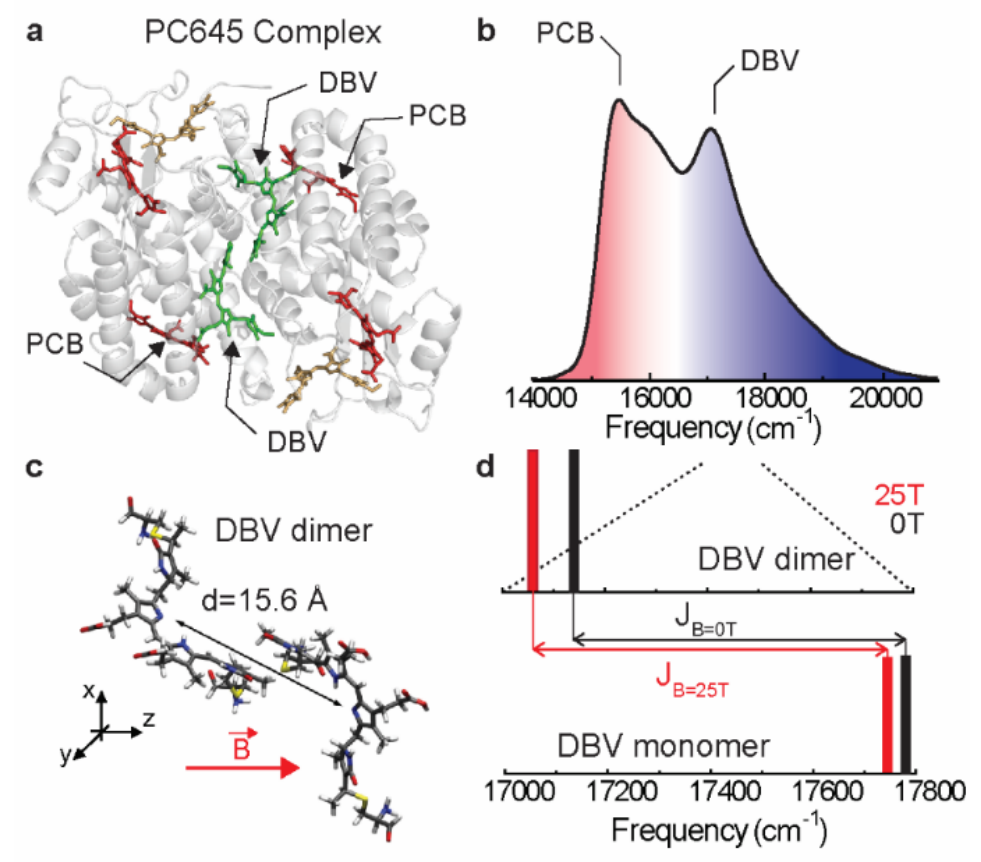

Figure 1. a, Pigment-protein arrangement of the PC645 light-harvesting complex. Four chromophore pairs are identified; of relevance to this study is the central DBV pair (green) and two pairs of PCB chromophores (red). b, Absorption spectrum of PC645 at 0 T. c, Molecular structure of the DBV dimer used for TDDFT modeling where the chromophores have a center-tocenter separation of $15.6 \AA$. d, Calculated excitation energies of the DBV monomer and dimer at $\mathrm{B}_{\mathrm{z}}=0 \mathrm{~T}$ (black) and $\mathrm{B}_{\mathrm{z}}=25 \mathrm{~T}$ (red). These values, Table 1, were used to calculate the shift of the electronic coupling induced by the magnetic field.

We applied a TDDFT method ${ }^{26-28}$ (details in Section 1 of the Supplementary Information, SI), to calculate the effect of a strong magnetic field on the excitonic coupling of the PC645 DBV 
dimer, (fig. 1c). We performed a series of calculations to predict the shift in the excitonic coupling at various magnetic field intensities, ranging from $0 \mathrm{~T}$ to $25 \mathrm{~T}$ (fig. S1). The calculated electronic excitation energies of the DBV monomer and dimer at $25 \mathrm{~T}$ are shown in figure $1 \mathrm{~d}$ and summarized in Table 1. Our calculations of the electronic coupling at zero field agree with previous quantumchemical calculations ${ }^{29}$.

Table 1: Comparison of the calculated electronic excitation energy $\left(\mathrm{E}, \mathrm{cm}^{-1}\right)$ and electronic coupling $\mathrm{J}$ of the DBV monomer and dimer at $\mathrm{B}_{\mathrm{z}}=0 \mathrm{~T}$ and $\mathrm{B}_{\mathrm{z}}=25 \mathrm{~T}$.

\begin{tabular}{ccc}
\hline & $\begin{array}{c}\mathrm{E}\left(\mathrm{B}_{\mathrm{z}}=0 \mathrm{~T}\right) \\
/ \mathrm{cm}^{-1}\end{array}$ & $\begin{array}{c}\mathrm{E}\left(\mathrm{B}_{\mathrm{z}}=25 \mathrm{~T}\right) \\
/ \mathrm{cm}^{-1}\end{array}$ \\
\hline Monomer & 17780 & 17744 \\
Dimer & 17140 & 17060 \\
$\mathrm{~J}$ & 640 & 684 \\
\hline$\Delta \mathrm{J}$ & \multicolumn{3}{c}{$\sim 44$} \\
\hline
\end{tabular}

The predicted spectral shifts induced on the DBV dimer by the magnetic field, however, are too small to be detected by steady-state absorption measurements, given the complexity and disorder within the light-harvesting complex that contribute to the broad lineshapes observed. Instead, we exploit femtosecond broadband pump-probe spectroscopy, which offers excellent sensitivity to quantum state splittings (vibrational or electronic) detected in the time domain and conveniently brought into the frequency domain by Fourier transformation.

\section{Broadband Pump-Probe at 25 T}

We utilized the Florida Split-Helix magnet ${ }^{30}$ developed at the National High Magnetic Field Laboratory (NHMFL) facility for generating the high magnetic fields used in these experiments. The Split-Helix is one of the largest magnets in the world specifically designed for advanced optical experiments at magnetic fields up to $25 \mathrm{~T}$. Our combined broadband pump- 
probe/Split-Helix system offers the unique capability to couple strong static magnetic fields with sub-20 fs laser pulses (details of the experimental apparatus in Section 2 of SI). Coherencesensitive experiments in such high magnetic fields are particularly challenging owing to the sometimes weak oscillatory signals and multiple sources of noise associated with generating such high magnetic fields (i.e. the temperature variation and the high water pressure needed to cool down the magnet during the experiment) which affects the signal-to-noise ratio of our measurements. Nevertheless, a test experiment at $25 \mathrm{~T}$ on a reference dye, cresyl violet, proves that high-sensitivity measurements are feasible The broadband laser pump stimulates vibrational wavepackets that are observed as oscillations imprinted superimposed on the pump-probe dynamics. By Fourier transforming the residual oscillations by a subtraction of the data with a biexponential fitting function, we retrieve the frequency response of the system. The strongest vibrational mode of cresyl violet has a frequency of $590 \mathrm{~cm}^{-1} 31$ and is unaffected by the $25 \mathrm{~T}$ field, supporting our hypothesis that strong magnetic fields do not alter nuclear motions (fig. S4).

To further validate our hypothesis, we also report a control study on a cryptophyte algae protein subunit containing a single PCB chromophore attached to the $\alpha$-subunit, which similarly show no changes in the ultrafast coherent response when the $25 \mathrm{~T}$ magnetic field is engaged (fig. S4). The control experiment on the subunit (fig. S5) reveals two intramolecular vibrations at 670 $\mathrm{cm}^{-1}$ and at $1630 \mathrm{~cm}^{-1}$. Details on the PC645 and $\alpha$-subunit sample preparation are described in Section 10 of SI.

Figure 2a shows the pump-probe differential transmission $(\Delta \mathrm{T} / \mathrm{T})$ map recorded for PC645 in solution at $25 \mathrm{~T}$ and ambient temperature. A similar measurement at $0 \mathrm{~T}$ is reported in the SI (fig. S5). The signal in both cases is dominated by instantaneous ground-state bleaching of the optically excited transitions of different chromophores in the protein. The $16800-17800 \mathrm{~cm}^{-1}$ 
probe range displays a sub-picosecond decaying component, assigned to the ultrafast energy transfer from the DVB donor state to the PCB acceptor excited states.
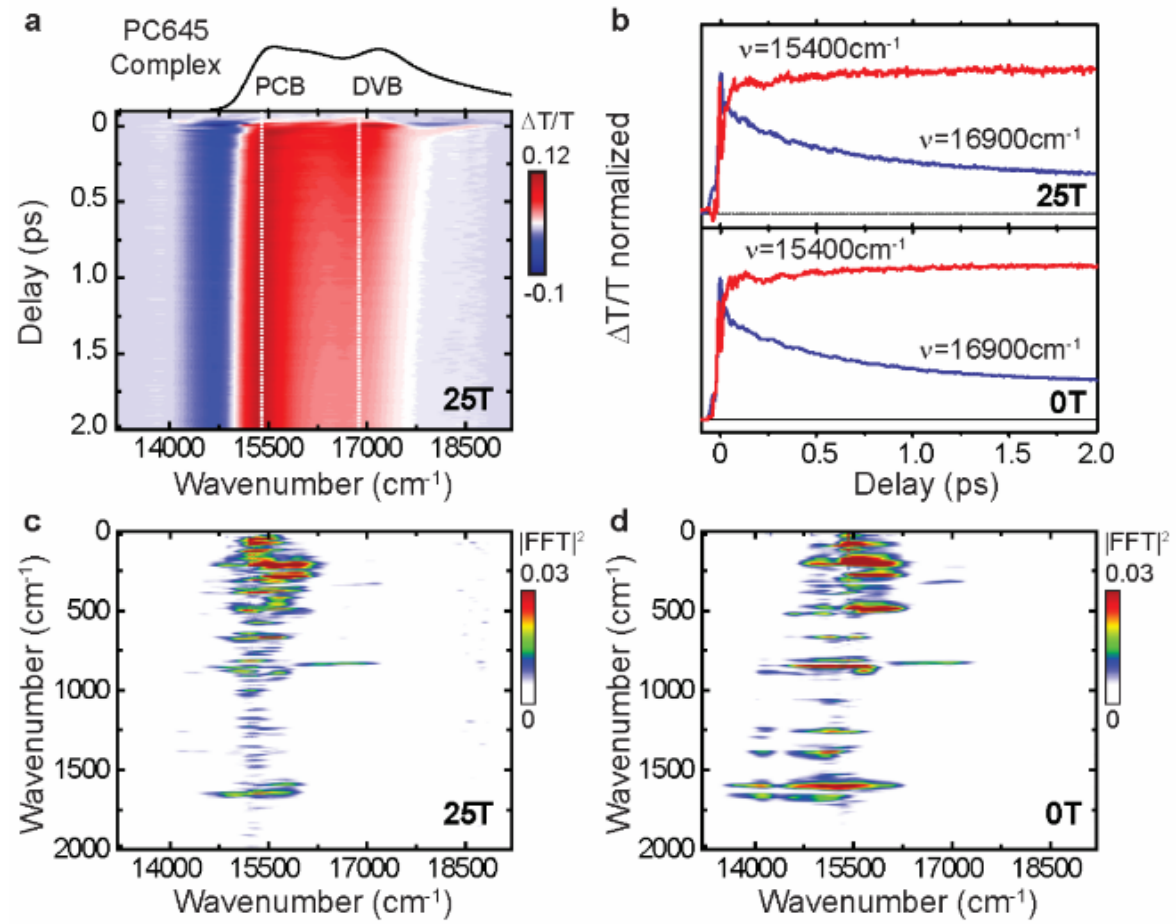

Figure 2. a, Pump-probe $\Delta \mathrm{T} / \mathrm{T}$ map of PC645 measured at $25 \mathrm{~T}$ and corresponding region of the linear absorption. b, selected pump-probe traces at DBV $\left(16900 \mathrm{~cm}^{-1}\right)$ and PCB $\left(15400 \mathrm{~cm}^{-1}\right)$ probe energies at $0 \mathrm{~T}$ and $25 \mathrm{~T}$. c, Fourier transform maps at $25 \mathrm{~T}$ and d, $0 \mathrm{~T}$.

Figure $2 \mathrm{~b}$ shows the decay of the DBV signature $\left(16900 \mathrm{~cm}^{-1}\right)$ and the corresponding growth of the PCB bleach signal $\left(15400 \mathrm{~cm}^{-1}\right)$ at both $0 \mathrm{~T}$ and $25 \mathrm{~T}$, which we assigned to the ultrafast energy transfer from the DVB donor state to the PCB acceptor excited states. The pumpprobe map of the PC645 complex taken at 0T is reported in figs. S6. We applied the same procedure described for the cresyl violet dye to obtain the Fourier transformed power maps at $25 \mathrm{~T}$ and 0T (figs. 2c and 2d). The clear pattern of coherences spanning from $200 \mathrm{~cm}^{-1}$ to $1700 \mathrm{~cm}^{-1}$, 
centered predominantly at the PCB region of the probe spectrum is preserved when the magnetic field is engaged.

In order to analyze the coherent signals at zero field and $25 \mathrm{~T}$, we integrated the power maps in figs. $2 \mathrm{c}$ and $2 \mathrm{~d}$ over all probe wavelengths. The results are reported in fig. 3 , where the 0 $\mathrm{T}$ and the $25 \mathrm{~T}$ power spectra measured at the NHMFL (blue and red curves, respectively) are displaced together with a reference spectrum (labeled as 'ref.', black curve in fig. 3) obtained from a similar pump-probe experiment on PC645 in a standard ultrafast laboratory.

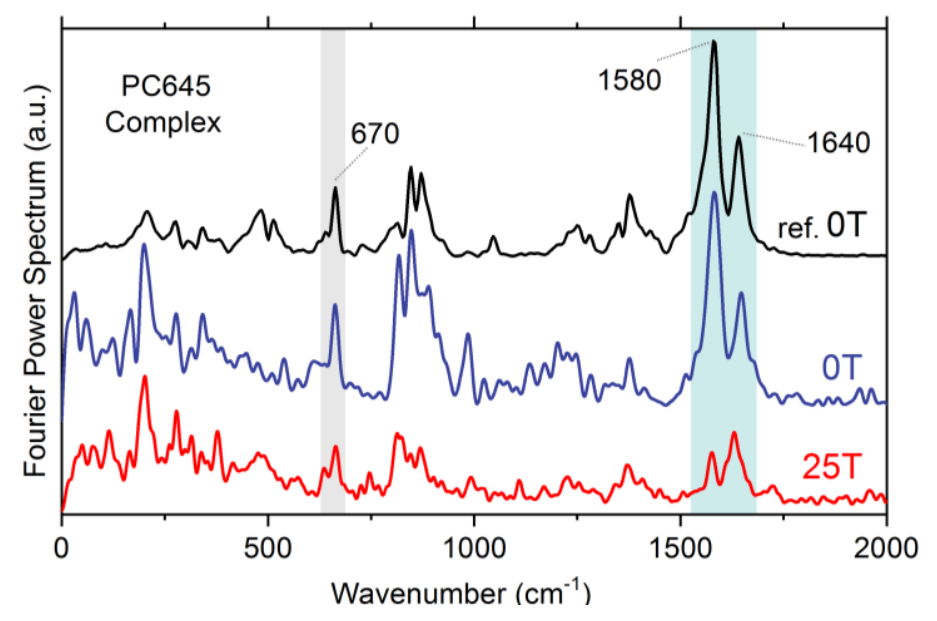

Figure 4. Integrated Fourier power spectra of PC645 at $25 \mathrm{~T}$ and $0 \mathrm{~T}$ (red and blue curves, respectively) obtained at the NHMFL and compared with the integrated Fourier power spectrum from a reference experiment at $0 \mathrm{~T}$ performed on the PC645 complex under the same experimental conditions at Princeton University laboratories. The shaded regions show signatures of the Fourier spectra which we focus on in this work, specifically, the lack of modulation of the $670 \mathrm{~cm}^{-1}$ vibrational mode, as well as the marked changes in the high frequency region between 1500 and $1700 \mathrm{~cm}^{-1}$. 
At $0 \mathrm{~T}$ strong coherent oscillations in the $200-350 \mathrm{~cm}^{-1}$ region, as well as distinct frequencies around $490 \mathrm{~cm}^{-1}, 670 \mathrm{~cm}^{-1}, 850 \mathrm{~cm}^{-1}, 1580 \mathrm{~cm}^{-1}$, and $1640 \mathrm{~cm}^{-1}$ are evident. While there are some clear differences in the $0 \mathrm{~T}$ experiment on PC645 at the NHMFL and the reference measurement, particularly at $800 \mathrm{~cm}^{-1}, 1000 \mathrm{~cm}^{-1}$, and in the low frequency region, we exclude changes in these frequency regions on the basis of setup-to-setup reproducibility, and instead focus on the power spectrum signatures which reproduce well the reference experiment, namely those at $675 \mathrm{~cm}^{-1}$ and between 1500 and $1700 \mathrm{~cm}^{-1}$. At these oscillation frequencies, the $0 \mathrm{~T}$ experiment on PC645 at NHMFL matches very well the reference measurement (blue and black curves in fig. 4), confirming the sensitivity at the NHMFL facility is adequate. The $25 \mathrm{~T}$ data (red curve in fig. 4) are largely consistent with the zero-field experiments: as expected, the spectral position of some of the largest peaks in the power spectrum are primarily unperturbed by the applied field, i.e. the position of the $670 \mathrm{~cm}^{-1}$ peak is preserved. However, a striking and reproducible change in the Fourier spectra intensity around $1580-1600 \mathrm{~cm}^{-1}$ is detected. Specifically, the amplitudes and locations of the $1580 \mathrm{~cm}^{-1}$ and $1640 \mathrm{~cm}^{-1}$ bands depend are somewhat sensitive to the strong magnetic field. Interestingly, the $1580 \mathrm{~cm}^{-1}$ frequency, previously assigned to a $\mathrm{C}=\mathrm{N}$ stretching mode of the PCB chromophore ${ }^{32-34}$, appears only as a small shoulder in the $\alpha$-subunit data (fig. S4).

We rationalize the amplitude difference of the $1580 \mathrm{~cm}^{-1}$ oscillation in the protein as a consequence of an amplification mechanism that becomes active when the chromophores interact closely due to their spatial arrangement in the protein scaffold. This hypothesis has been recently described on the basis of 2DES studies on the PC645 complex ${ }^{34}$. It was proposed that the vibrational coherence experiences a coherent amplification through vibronic coupling between the energetically remote DBV and PCB chromophores, due to a resonance of the 
intramolecular PCB vibration with the electronic energy gap between the lower excitonic $\mathrm{DVB}^{-}$ state and the excited PCB state ${ }^{35}$. The response of these oscillations to the magnetic field suggests that the shift of the excitonic $\mathrm{DBV}^{-}$band could be affecting those coherences via changes in vibronic coupling to $\mathrm{PCB}$.

\section{Vibronic Model}

We use a bare-bones vibronic coupling model to qualitatively predict how the coherence amplitudes of vibrations of the PCB chromophores are perturbed by mixing with the electronic DBV$^{-}$state (for details see Section 7 in SI). In our model (fig. 4a) we consider: the DBV־ exciton state, the PCB excited state and two local PCB vibrations, at $v_{1}=1560 \mathrm{~cm}^{-1}$ and $v_{2}=1640 \mathrm{~cm}^{-1}$. We do not include the DBV vibrations in the model as those vibrationally excited states are not close enough in energy to the PCB acceptor vibrational ground state for appreciable FranckCondon overlap.

The coupling between these the quasi- resonant DBV exciton and PCB vibrationally excited $\mathrm{S}_{1}$ leads to two vibronic eigenstates $\left(v_{1}^{*}\right.$ and $\left.v_{2}{ }^{*}\right)$ in the relevant energy window. The degree of vibronic mixing depends on: (i) the detuning between the vibrational frequency and the electronic energy gap $\Delta \mathrm{E}$ (fixed at $1560 \mathrm{~cm}^{-1}$ in resonance with $v_{1}$ ); (ii) the Huang-Rhys factor of the vibrational modes $(\mathrm{S}=0.08)$, and (iii) the electronic coupling between the DBV exciton state and PCB $\left(\mathrm{J}_{\mathrm{PCB} / \mathrm{DBV}}=44 \mathrm{~cm}^{-1}\right.$ at $0 \mathrm{~T}$ and $\mathrm{J}_{\mathrm{PCB} / \mathrm{DBV}}=48 \mathrm{~cm}^{-1}$ at $25 \mathrm{~T}$, as calculated in Section 6 in $\mathrm{SI})$.

In order to compare our experimental trend with simulations based on our hypothesized model, Fig $4 \mathrm{~b}$ (vide infra), we fit the frequency-domain filtered oscillations, recorded at the various magnetic field strengths, in the time domain with a sum of two damped cosines. The 
fitting results are summarized in Table S2 in SI. The relative amplitudes of the $1580 \mathrm{~cm}^{-1}$ versus $1640 \mathrm{~cm}^{-1}$ oscillations are clearly magnetic field dependent, Fig 4c.
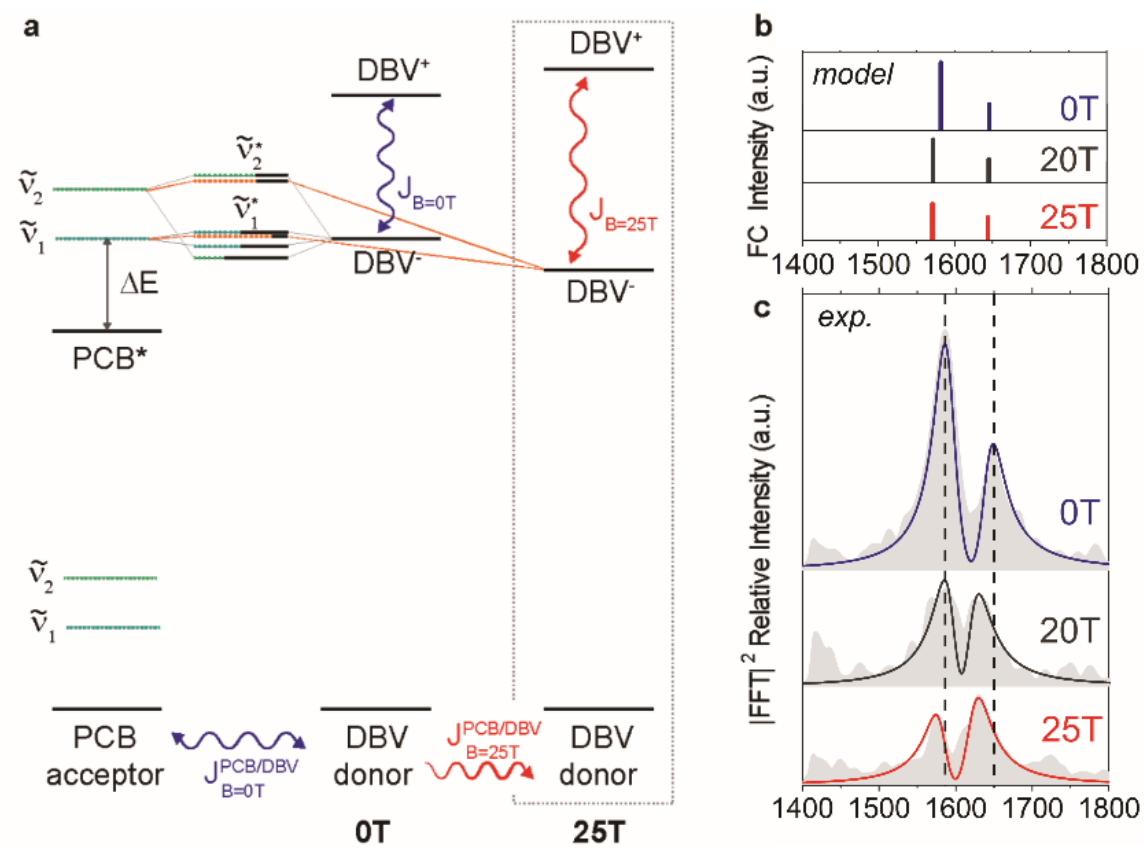

Figure 4. a, Energy level schemes of the vibronically coupled DBV/PCB chromophores. Solid black lines represent electronic states, while the dotted green lines represent the vibrational states of the PCB; vibronic states are labeled as $v^{*} . \mathrm{J}_{\mathrm{B}}$ is the electronic coupling between the two DBV excitonic states; $\mathrm{J}^{\mathrm{PCB} / \mathrm{DBV}}$ is the electronic coupling between the DBV and the PCB chromophore, and it weakly depends on the magnetic field. b, Simulated amplitudes of bands associated with the two oscillations $v_{1} *$ and $v_{2} *$ at $0 \mathrm{~T}, 20 \mathrm{~T}$, and $25 \mathrm{~T}$., obtained from summation of the amplitudes associated with all Feynman pathways contributing to each coherence ${ }^{34}$ (details discussed in Section 9 in the SI). c, Expansion of measured integrated Fourier power spectra (gray shadows) in the high frequency spectral region at $0 \mathrm{~T}, 20 \mathrm{~T}$ and $25 \mathrm{~T}$. Solid lines are obtained by fitting the filtered oscillations with the sum of two damped cosines. 
At zero field we found that vibronic mixing of the $\mathrm{DBV}^{-}$with the two $\mathrm{PCBs}$ yields states with a strong intensity at $1580 \mathrm{~cm}^{-1}$ and a less intense band at $1645 \mathrm{~cm}^{-1}$ (black bars in fig. $4 \mathrm{~b}$ ). When we simulated the effect of the $25 \mathrm{~T}$ field tuning the excitonic $\mathrm{DBV}^{-}$state away from resonance (changing $\mathrm{J}_{\mathrm{B}}$ by $\sim 44 \mathrm{~cm}^{-1}$ ), we found a significant change in the lower vibronic eigenstate. Both the vibronic bands undergo a small red shift (by $\sim 10 \mathrm{~cm}^{-1}$ ) (red bars in fig. $4 \mathrm{~b}$ ), and the intensity of the $1580 \mathrm{~cm}^{-1}$ band measured at $0 \mathrm{~T}$ is strongly decreased at $25 \mathrm{~T}$. The amplitude of the $1640 \mathrm{~cm}^{-1}$ is much less sensitive to the excitonic detuning, because of the larger detuning $\Delta \mathrm{E}$ compared to the vibrational frequency $v_{2}$, although its slight redshift is captured well by our model. This interpretation is supported by the pump-probe data recorded at an intermediate magnetic field of $20 \mathrm{~T}$ (fig. S6) and further highlights the favorable resonance conditions of the $1580 \mathrm{~cm}^{-1}$ vibrational mode at ambient conditions. Our calculations are in reasonable agreement with the trend evident in experimental results reported in fig. $4 \mathrm{c}$, which shows an expanded view of the power spectra described in fig. 4 for the $0 \mathrm{~T}$ and the $25 \mathrm{~T}$, together with the results at $20 \mathrm{~T}$.

We have found that a very strong magnetic field can perturb the excitonic electronicvibrational resonances of electronically coupled chromophores in diamagnetic systems. For the first time we have combined an intense static high magnetic field with ultrafast broadband spectroscopy to inspect vibronic coherences in a photosynthetic complex. We find that the vibronic tuning markedly affects the amplitude of the $1580 \mathrm{~cm}^{-1}$ band, although we note that the detuning at high field strengths of this vibronic resonance does not affect the energy transfer rate, as shown in fig. S8. It is possible, though, that the vibronic resonance plays a role in guiding excitation energy by biasing the transfer of energy to two of the four PCB acceptor molecules. We rationalize, then, that while the overall interchromophoric coupling is not sensitive to these 
vibronic resonances, the appearance of this type of sensitivity to the electronic-vibrational resonance condition may serve as an indicator of vibrationally-assisted energy transfer, as discussed in ref. 35. Nevertheless, this unique magnetic field effect helps reveal the complex network of couplings responsible for not only driving energy transfer among pigments within the protein but also tuning the observed frequencies and intensities of coherences in the experiment. These are built firstly from the excitonic coupling of the DBV donor pair, which subsequently affects the downstream vibronic coupling with the PCB acceptors.

Our work reveals the sensitivity of vibronic coupling to electronic-vibrational resonance as the field shifts the electronic donor state relative to the acceptor vibrational states. This result indicates that controlled chromophore-chromophore interactions for light harvesting may involve a surprisingly precise balance of energy gaps. This approach may contribute to framing the mechanisms of vibronically assisted photo-induced dynamics and use them to illuminate design principles for directed light-harvesting.

\section{ACKNOWLEDGMENT}

The authors acknowledge financial support by National Science Foundation, MRI No. DMR1229217 and by Princeton University through the Innovation Fund for New Ideas in the Natural Sciences. A portion of this work was performed at the National High Magnetic Field Laboratory, which is supported by National Science Foundation Cooperative Agreement No. DMR-1157490 and the State of Florida. M.M. acknowledges financial support by European Community (H2020 Marie Skłodowska-Curie Actions), Project No. 655059. M. B. O. acknowledges financial support by the National Science Foundation - Institute for Complex Adaptive Matter (NSF- 
ICAM) and the National Science Foundation for the use of supercomputing resources through the Extreme Science and Engineering Discovery Environment (XSEDE), Project No. TGCHE140097. B.K acknowledges support by the National Science Foundation Graduate Research Fellowship under Grant Number DGE-1656466. M. M., J. C. D., M. B. and S. MG. acknowledge Dmitry Semenov for the valuable support during the magnet time.

\section{REFERENCES}

(1) Chenu, A., Scholes, G. D. Coherence in Energy Transfer and Photosynthesis. Annu. Rev. Phys. Chem. 2015, 66, 69-96, DOI:10.1146/annurev-physchem-040214-121713.

(2) Engel, G. S., Calhoun, T. R., Read, E.L., Ahn, T.K., Mančal, T., Cheng, Y-C., Blankenship, R.E., Fleming, G.R. Evidence for Wavelike Energy Transfer through Quantum Coherence in Photosynthetic Systems. Nature. 2007, 446, 782-786, DOI:10.1038/nature05678.

(3) Collini, E., Wong, C.Y., Wilk, K.E., Curmi, P.M.G., Brumer,P., Scholes, G.D. Coherently Wired Light-harvesting in Photosynthetic Marine Algae at Ambient Temperature. Nature. 2010, 463, 644-647, DOI:10.1038/nature08811.

(4) Panitchayangkoon, Hayes, G., Fransted, K.A., Caram, J.R., Harel, E., Wen, J., Blankenship, R.E., Engel, G.S. Long-lived Quantum coherence in photosynthetic complexes at physiological temperature. Proc. Natl. Acad. Sci. USA. 2010, 107, 12766-12770, DOI:10.1073/pnas.1005484107. 
(5) Mirkovic, T., Ostroumov, E.E., Anna, J.M., van Grondelle, R., Govindjee, Scholes, G.D. Light Absorption and Energy Transfer in the Antenna Complexes of Photosynthetic Organisms. Chem. Rev. 2016, DOI:10.1021/acs.chemrev.6b00002.

(6) Christensson, N., Kauffmann, H. F., Pullerits, T. \& Mancal, T. Origin of Long-Lived Coherences in Light-harvesting Complexes. J. Phys. Chem. B. 2012, 116, 7449-7454, DOI:10.1021/jp304649c.

(7) Kolli, A., O'Reilly, E. J., Scholes, G. D. \& Olaya-Castro, A. The Fundamental Role of Quantized Vibrations in Coherent Light Harvesting by Cryptophyte Algae. J. Chem. Phys. 2012, 137, 174109, DOI:10.1063/1.4764100.

(8) Chenu, A., Christensson, N., Kauffmann, H. F. \& Mancal, T. Enhancement of Vibronic and Ground-state Vibrational Coherences in 2D Spectra of Photosynthetic Complexes. Sci. Rep. 2013, 3, 2029, DOI:10.1038/srep02029.

(9) Tiwari, V., Peters, W. K. \& Jonas, D. M. Electronic Resonance with Anticorrelated Pigment Vibrations Drives Photosynthetic Energy Transfer Outside the Adiabatic Framework. P. Natl. Acad. Sci. USA. 2013, 110, 1203-1208, DOI:10.1073/pnas.1211157110.

(10) Chin, A. W., Prior, J., Rosenbach, R., Caycedo-Soler, F., Huelga, S.F., Plenio, M.B. The Role of Non-equilibrium Vibrational Structures in Electronic Coherence and Recoherence in Pigment-Protein Complexes. Nat. Phys. 2013, 9, 113-118, DOI:10.1038/NPHYS2515.

(11) Plenio, M. B., Almeida, J. \& Huelga, S. F. Origin of Long-lived Oscillations in 2Dspectra of a Quantum Vibronic Model: Electronic Versus Vibrational Coherence. J. Chem. Phys. 2013, 139, 235102, DOI:10.1063/1.4846275. 
(12) Lim, J., Paleček, D., Caycedo-Soler, F., Lincoln, C.N., Prior, J., von Berlepsch, H., Huelga, S.F., Plenio, M.B., Zigmantas D., Hauer, J. Vibronic Origin of Long-lived Coherence in an Artificial Molecular Light Harvester. Nat. Commun. 2015, 6, DOI:10.1038/ncomms8755.

(13) Chin, A. W., Datta, A., Caruso, F., Huelga, S. F. \& Plenio, M. B. Noise-assisted Energy Transfer in Quantum Networks and Light-harvesting Complexes. New J. Phys. 2010, 12, 065002, DOI:10.1088/1367-2630/12/6/065002.

(14) Womick, J. M. \& Moran, A. M. Vibronic Enhancement of Exciton Sizes and Energy Transport in Photosynthetic Complexes. J. Phys. Chem. B. 2011, 115, 1347-1356, DOI:10.1021/jp106713q.

(15) Prior, J., Chin, A. W., Huelga, S. F. \& Plenio, M. B. Efficient Simulation of Strong System-Environment Interactions. Phys. Rev. Lett. 2010, 105, 050404, DOI: 10.1103/PhysRevLett.105.050404.

(16) Romero, E., Augulis, R., Novoderezhkin, V.I., Ferretti, M., Thieme, J., Zigmantas D., van Grondelle, R. Quantum Coherence in Photosynthesis for Efficient Solar-energy Conversion. Nat. Phys. 2014, 10, 677-683, DOI:10.1038/Nphys3017.

(17) Freed, K. F. in Radiationless Processes in Molecules and Condensed Phases: in Molecules and Condensed Phases. 23-168 (Springer Berlin Heidelberg, 1976).

(18) Zhang, Y., Liu, C., Balaeff, A., Skourtis, S. S. \& Beratan, D. N. Biological Charge Transfer via Flickering Resonance. P. Natl. Acad. Sci. USA. 2014, 111, 10049-10054, DOI:10.1073/pnas.1316519111. 
(20) Jordan, M. W., Brantley, A. W., Norbert, F. S. \& Andrew, M. M. Vibronic Effects in the Spectroscopy and Dynamics of C-phycocyanin. J. Phys. B. 2012, 45, 154016, DOI:10.1088/0953-4075/45/15/154016.

(21) Fuller, F. D. Pan, J., Gelzinis, A., Butkus, V., Senlik, S.S., Wilcox, D.E., Yocum, C.F., Valkunas, L., Abramavicius D., Ogilvie, J.P. Vibronic Coherence in Oxygenic Photosynthesis. Nat. Chem. 2014, 6, 706-711, DOI:10.1038/Nchem.2005.

(22) Fowler, G. J. S., Visschers, R. W., Grief, G. G., van Grondelle, R., Hunter, C. N. Genetically Modified Photosynthetic Antenna Complexes with Blueshifted Absorbency Bands. Nature. 1992, 355, 848-850, DOI:10.1038/355848a0.

(23) Canters, G. W. \& Van der Waals, J. H. The Porphyrins. 531-582 (Academic Press: New York, 1978).

(24) Marin, A. Doust, A.B., Scholes, G.D., Wilk, K.E., Curmi, P.M.G., van Stokkum, I.H.M., van Grondelle, R. Flow of Excitation Energy in the Cryptophyte Light-Harvesting Antenna Phycocyanin 645. Biophys. J. 2011, 101, 1004-1013,

DOI:10.1016/j.bpj.2011.07.012.

(25) Mirkovic, T., Doust, A.B., Kim, J., Wilk, K.E., Curutchet, C., Mennucci, B., Cammi, R., Curmi, P.M.G, Scholes, G.D.Ultrafast Light Harvesting Dynamics in the Cryptophyte Phycocyanin 645. Photochem. Photobio. Sci. 2007, 6, 964-975, DOI:10.1039/b704962e.

(26) Andrade, X. Strubbe, D., De Giovannini, U., Larsen, A.H., Oliveira, M.J., AlberdiRodriguez, J., Varas A., Theophilou, I., Helbig N., Verstraete, M.J., Stella, L., Nogueira, F., Aspuru-Guzik, A., Castro A., Marques, M.A., Rubio, A. Real-space Grids and the Octopus 
Code as Tools for the Development of New Simulation Approaches for Electronic Systems. Phys. Chem. Chem. Phys. 2015, 17, 31371-31396, DOI:10.1039/c5cp00351b.

(27) Marques, M. A. L., Castro, A., Bertsch, G. F. \& Rubio, A. Octopus: a First-principles Tool for Excited Electron-ion Dynamics. Comput. Phys Commun. 2003, 151, 60-78, DOI:10.1016/S0010-4655(02)00686-0.

(28) Castro, A., Appel, H., Oliveira, M., Rozzi, C.A., Andrade, X., Lorenzen, F., Marques, M.A.L., Gross, E.K.U., Rubio, A. Octopus: a Tool for the Application of Time-dependent Density Functional Theory. Phys. Status Solidi B. 2006, 243, 2465-2488, DOI:10.1002/pssb.200642067.

(29) Harrop, S. J., Wilk, K.E., Dinshaw, R., Collini, E., Mirkovic, T., Teng, C.Y., Oblinsky, D.G., Green, B.R., Hoef-Emden, K., Hiller, R.G., Scholes, G.D., Curmi, P.M.G. Singleresidue Insertion Switches the Quaternary Structure and Exciton States of Cryptophyte Lightharvesting Proteins. Proc. Natl. Acad. Sci. USA. 2014, 111, E2666-2675, DOI:10.1073/pnas.1402538111.

(30) Toth, J. \& Bird, M. D. FEA-Aided Design for a Working Model of a Split Florida-Helix. IEEE Transactions on Applied Superconductivity. 2008, 18, 575-578, DOI:10.1109/TASC.2008.921942.

(31) Fuji, T., Saito, T. \& Kobayashi, T. Dynamical Observation of Duschinsky Rotation by Sub-5-fs Real-time Spectroscopy. Chem. Phys. Lett. 2000, 332, 324-330, DOI:10.1016/S0009-2614(00)01278-1. 
(32) Arpin, P. C., Turner, D.B., McClure, S.D., Jumper, C.C., Mirkovic, T., Challa, J.R., Lee, J., Teng, C.Y., Green, B.R., Wilk, K.E., Curmi, P.M.G., Hoef-Emden, K., McCamant, D.W., Scholes, G.D., Spectroscopic Studies of Cryptophyte Light Harvesting Proteins: Vibrations and Coherent Oscillations. J. Phys. Chem. B. 2015, 119, 10025-10034, DOI:10.1021/acs.jpcb.5b04704.

(33) Kneip, C., Parbel, A., Foerstendorf, H., Scheer, H., Siebert, F., Hildebrandt, P. Fourier Transform Near-infrared Resonance Raman Spectroscopic Study of the Alpha-subunit of Phycoerythrocyanin and Phycocyanin from the Cyanobacterium Mastigocladus Laminosus. J. Raman. Spectrosc. 1998, 29, 939-944, DOI:10.1002/(Sici)10974555(199810/11)29:10/11<939::Aid-Jrs320>3.0.Co;2-X.

(34) Andel, F., Murphy, J.T., Haas, J.A., McDowell, M.T., van der Hoef, I., Lugtenburg, J., Lagarias, J.C., Mathies, R.A. Probing the Photoreaction Mechanism of Phytochrome through Analysis of Resonance Raman Vibrational Spectra of Recombinant Analogues.

Biochemistry. 2000, 39, 2667-2676, DOI:10.1021/bi991688z.

(35) Dean, J. C., Mirkovic, T., Toa, Z. S. D., Oblinsky, D. G. \& Scholes, G. D. Vibronic Enhancement of Algae Light Harvesting. Chem. 2016, 1, 858-872,

DOI:10.1016/j.chempr.2016.11.002. 